\title{
UPREGULATED EXPRESSION OF ONCOMODULIN, THE BETA ISOFORM OF PARVALBUMIN, IN PERIKARYA AND AXONS IN THE DIENCEPHALON OF PARVALBUMIN KNOCKOUT MICE
}

\author{
B. CSILLIK, ${ }^{\text {a* }}$ B. SCHWALLER, ${ }^{\text {b1 }}$ A. MIHALY, ${ }^{\text {a2 }}$ \\ T. HENZI, ${ }^{\text {b1 }}$ E. LOSONCZI ${ }^{\mathrm{c} 3}$ AND E. KNYIHAR-CSILLIK ${ }^{\mathrm{c2}}$ \\ ${ }^{a}$ Department of Anatomy, Albert Szent-Györgyi Medical School, Uni- \\ versity of Szeged, Hungary \\ ${ }^{b}$ Unit of Anatomy, Department of Medicine, University of Fribourg, \\ Switzerland \\ ${ }^{c}$ Department of Neurology, Albert Szent-Györgyi Medical School, Uni- \\ versity of Szeged, Hungary
}

\begin{abstract}
The calcium-binding proteins parvalbumin, calbindin D-28k, calretinin and calcineurin are present in subsets of GABAergic gigantic calyciform presynaptic terminals of the reticular thalamic nucleus (RTN). Previously it was hypothesized that GABA and calcium-binding proteins including parvalbumin are not only colocalized in the same neuron subpopulation, but that GABA synthesis and parvalbumin expression could be also genetically regulated by a common mechanism. Moreover, parvalbumin expression levels could influence GABA synthesis. For this, we analyzed GABA immunoreactivity in RTN gigantic calyciform presynaptic terminals of parvalbumin-deficient (PV-I-) mice. With respect to GABA immunoreactivity we found no differences compared to wild-type animals. However, using a polyclonal parvalbumin antibody raised against full-length rat muscle parvalbumin on brain sections of PV $-I-$ mice, we observed paradoxical parvalbumin immunoreactivity in partly varicose axons in the diencephalon, mainly in the lamina medullaris externa surrounding the thalamus. A detailed immunohistochemical, biochemical and molecular biological analysis revealed this immunoreactivity to be the result of an upregulation of oncomodulin (OM), the mammalian beta isoform of parvalbumin in PV-I- mice. In addition, OM was present in a sparse subpopulation of neurons in the thalamus and in the dentate gyrus. OM expression has not been observed before in neurons of the mammalian brain; its expression was restricted to outer hair cells in the organ of Corti. Our results indicate that the absence of parvalbumin has no major effect on the GABA-synthesizing system in RTN presynaptic terminals excluding a direct effect of parvalbumin on this regulation. However, a likely homeostatic mechanism is induced resulting in the upregulation of $\mathrm{OM}$ in selected axons and neuronal

${ }^{1}$ Present address: Abteilung Anatomie, Department Medizin, Universität Fribourg, Route Albert-Gockel 1, CH-1700 Fribourg, Switzerland.

${ }^{2}$ Present address: Department of Anatomy, University Medical School, 40 Kossuth Lajos sgt, H-6701 Szeged, Hungary.

${ }^{3}$ Present address: Richter Gedeon NYRT, Gyomroi ut 19-21, Budapest 1103, Hungary.

${ }^{*}$ Correspondence to: B. Csillik, Department of Anatomy, University Medical School, 40 Kossuth Lajos sgt, H-6701 Szeged, Hungary. Tel: +36-62-544-918; fax: +36-62-545-707.

E-mail address: csillik@anatomy.szote.u-szeged.hu (B. Csillik).

Abbreviations: CaBP, calcium-binding proteins; OM, oncomodulin;

PAGE, polyacrylamide gel electrophoresis; PBS, phosphate-buffered saline; PV, parvalbumin; PV-I-, parvalbumin-deficient; RTN, reticular
\end{abstract} thalamic nucleus; SDS, sodium dodecyl sulphate. perikarya. Our results warrant further detailed investigations on the putative role of $O M$ in the brain.

Key words: reticular thalamic nucleus, thalamus, GABA, parvalbumin, oncomodulin, regeneration.

At the level of gross anatomy, the reticular thalamic nucleus (RTN) is a crescent-like structure, similar to a halfeggshell, surrounding the lateral, superior and inferior aspects of the thalamus (Paxinos and Watson, 1982; Sidman et al., 1971). The GABAergic RTN is a heterogeneous structure, from cytoarchitectural, immunocytochemical, electrophysiological and neuropharmacological viewpoints alike (Steriade, 2001). It occupies a strategic position between specific nuclei of the thalamus and the cerebral cortex (Steriade, 2001; Pinault, 2004), as a preferential target of corticothalamic projections, located at the intersection of thalamo-cortical and cortico-thalamic pathways. The RTN is implied in almost all the functional modalities represented by its motor, somatosensory, visceral, auditory, gustatory and limbic sectors (Shosaku et al., 1989). In addition, the RTN plays an important part in transforming nociception into pain (Gauriau and Bernard, 2002; KnyiharCsillik and Csillik, 2006), by regulating attention and distraction to potentially painful stimuli, functioning as a coincidence detector (Kilmer, 2001). It has been shown by electrophysiological and immunohistochemical studies by Knyihar-Csillik et al. (2005) that a two-way traffic between RTN and the retrosplenial cortex is involved in the communication between RTN and cerebral cortex.

In the large GABAergic calyciform presynaptic terminals of RTN of the rat, several calcium-binding proteins (CaBP) including parvalbumin (PV), calbindin D-28k, calretinin and calcineurin have been observed (Csillik et al., 2002a,b, 2004, 2005, 2006). At the light microscopic level, RTN is characterized by an intense PV immunoreaction, which originally was exclusively attributed to PV-immunoreactive neurons (Celio, 1990). Recent light- and electron histochemical studies revealed however that some of the structures, erroneously identified as nuclei, are in reality cross-sections of large dendrites, while the PV-immunoreactive structures thought to correspond to nerve cell perikarya, are in reality presynaptic dendraxonic terminals (Csillik et al., 2002a, 2004). The PV-immunoreactive calyciform presynaptic terminals contain GABA (Csillik et al., $2005,2006)$. This raised the question whether the GABAsynthesizing enzyme GAD and calcium-binding proteins, in particular the one most often present in these terminals, 
i.e. PV, are not only co-expressed in the same neuron subpopulation, but whether they are also genetically regulated by a common mechanism. Furthermore, the observation that $P V$ is almost exclusively expressed in GABAergic neurons suggested that PV directly or indirectly could affect the type or amount of neurotransmitter in the PVimmunoreactive neuron population. In the cortex of PV - /mice, differences in the firing properties of pyramidal cells suggested that $\mathrm{PV}$ plays a key role in the regulation of local inhibitory effects exerted by GABAergic interneurons on pyramidal neurons (Schwaller et al., 2004, MCN). Furthermore, analysis of non-linear coupling of local field potentials indicated that absence of PV not only affects local neuronal networks within the cortex, but might also affect interacting sites that are distant from the recording electrodes but close to each other, e.g. within the thalamus (Villa et al., 2000). Thus, we decided to study by immunohistochemistry the distribution of CaBPs in the RTN of PV knockout (PV-I-) animals. Unexpectedly, in brain sections from $\mathrm{PV}-/-$ mice stained with one particular polyclonal anti-PV antiserum produced against full-length rat PV, "PV-immunoreactive" structures were detected. Both, structural details of these immunopositive entities and the identity of the molecule giving rise to this paradoxical PV staining, oncomodulin (OM), the beta isoform of $\mathrm{PV}$, are described in this report.

\section{EXPERIMENTAL PROCEDURES}

In these studies, the number of animals (rats/mice) was kept to a minimum and efforts were taken to minimize the suffering; young adult Wistar rats, young adult male mice of the C57BI/6J strain and homozygous parvalbumin-knockout (PV-l-) mice (Schwaller et al., 1999; Vecellio et al., 2000) were analyzed. As compared to the initial strain reported before (Schwaller et al., 1999) with a mixed 129Ola Hsd $\times$ C57BI/6J genetic background, the animals used in this study had been backcrossed to $\mathrm{C} 57 \mathrm{BI} / 6 \mathrm{~J}$ animals for at least 10 generations and are thus considered to be congenic with C57BI/6J mice (new name C57PV-/-). For the genotyping, genomic DNA was isolated from tail biopsies that was followed by PCR using primer pairs that were either specific for exon 3 (deleted in $\mathrm{PV}-/-$ mice) or for part of the neomycin resistance cassette (absent in PV $+/+$ mice). All mice were housed in groups before use; they were adult (25-30 g) when used for experiments. Care of the animals complied with the guidelines of the Hungarian Ministry of Welfare and was in accordance with the European Communities Council Directive (November 24, 1986; 86/609/ EEC), the NIH Guide for the Care and Use of Laboratory Animals (NIH Publications No. 85-23, revised 1985) and the Guidelines for Ethics in Animal Experiments, University of Szeged, Albert SzentGyörgyi Medical School. After an i.p. injection with a lethal dose of chloral hydrate, the animals were subjected to transcardial fixation with $4 \%$ formaldehyde, $0.5 \%$ glutaraldehyde in phosphate-buffered saline (PBS). Brains were removed and processed in an ascending series of sucrose, containing $4 \%$ formaldehyde.

\section{PV immunohistochemistry}

Serial cryostat sections, $20 \mu \mathrm{m}$ thick, were obtained in the paramedial plane, comprising a thickness of $1-3 \mathrm{~mm}$ as measured from the midsagittal plane. RTN was localized in mice, using the parameters of the Sidman-Angevine-Pierce stereotactic atlas 1971, and in rats, using the Paxinos-Watson atlas (1982). On free-floating sections or in sections adhered to Superfrost UltraPlus specimen holders (Menzel, Braunschweig, Germany), PV was detected immunohistochemically in rats and mice, by using a rabbit polyclonal anti-mouse $\mathrm{PV}$ antibody raised against full-length PV from rat (sc-7449, Santa Cruz, CA, USA) Preliminary experiments showed best staining results with antibody dilutions of 1:2000-1:3500. Endogenous peroxidase activity was blocked by $0.3 \%$ hydrogen peroxide diluted in methanol, for $10 \mathrm{~min}$, followed by three successive rinses in $0.1 \mathrm{M}$ phosphate buffer, $\mathrm{pH} 7.4$. Free-floating or adherent sections were pre-treated with blocking serum (0.1-1.0 M PBS, 10\% normal goat serum, 1\% bovine serum albumin (BSA) and $0.3 \%$ Triton $\mathrm{X}-100$ ) on a shaker plate at room temperature for $1 \mathrm{~h}$, and then transferred into a solution containing the primary antibody. Incubation was carried out at $4{ }^{\circ} \mathrm{C}$ on a shaker for $36 \mathrm{~h}$, followed by three rinses in $0.1 \mathrm{M}$ phosphate buffer. To detect the bound primary antibody, we used the avidinbiotin-peroxidase method. Kits were obtained from Vector Laboratories (Burlingame, CA, USA). The secondary antibody, biotinylated anti-rabbit immunoglobulin was applied for $90 \mathrm{~min}$ at room temperature. Three more rinses in $0.1 \mathrm{M}$ phosphate buffer were followed by incubation with the avidin-biotinylated peroxidase complex for $60 \mathrm{~min}$ at room temperature. After three rinses in 0.1 $M$ phosphate buffer, peroxidase activity was visualized by the histochemical reaction involving diamino-benzidine-tetrahydrochloride (DAB) and hydrogen peroxide $\left(3 \mu \mathrm{l}\right.$ of $30 \% \mathrm{H}_{2} \mathrm{O}_{2}$ in $10 \mathrm{ml}$ $1 \% \mathrm{DAB})$. After three rinses in $0.1 \mathrm{M}$ phosphate buffer, the sections were dehydrated in a graded series of alcohol, cleared in xylene and coverslipped with Permount.

\section{OM immunohistochemistry}

An identical protocol was used for the detection of OM, the mammalian beta isoform of parvalbumin (MacManus, 1979), but as the first antibody the affinity-purified polyclonal goat anti-OM antibody (PV-beta, N-19, Santa Cruz Biotechnology, Inc., Santa Cruz, CA, USA) was used. This peptide antibody was raised against a region in the N-terminus of human OM showing very low homology to human PV. For control experiments, the blocking peptide sc7446P (Santa Cruz) was applied. Alternatively, the polyclonal rabbit anti-OM antiserum OM3 from Swant (Bellinzona, Switzerland) raised against recombinant full-length rat OM was used. Staining of sections with both sera yielded identical results. For all antibodies, a series of experiments were carried out to demonstrate the specificity of the antibody and included: (1) omission of the first specific antiserum; (2) incubation with normal rabbit or mouse serum instead of incubation with the anti-PV or anti-OM antibodies; (3) treatment according to the avidin-biotin complex method, from which one of the steps had been omitted and (4) preadsorption of the specific antibody with blocking peptides. None of the specimens treated by one of the above methods showed any specific immunoreactivity.

\section{GABA immunoreactivity}

GABA immunoreactivity was detected, applying the same protocol but using a rabbit anti-GABA antiserum (Chemicon, Temecula, CA, USA) at a dilution of 1:500.

\section{Stereology}

The number of calyciform terminals was determined in consecutive coronal section series according to the method of West et al. (1991), using a $100 \times$ oil immersion lens. Analysis was performed with the aid of an Olympus microscope (Olympus, Tokyo, Japan) equipped with a video camera and stepping motors. Counting frames were superimposed on video images of the microscopic fields.

\section{Western blot analysis}

Western blot analysis was performed on tissue from the diencephalons of brains of adult PV $-/-$ mice, according to the protocol of Kamps and Sefton (1988), modified by Rogers et al. (1991) as 
follows. The tissue was homogenized with a Disperser T10 Basic (IKA-Werke, Oberkochen, Germany) in $50 \mathrm{mM}$ Tris buffer ( $\mathrm{pH} 7.5$ ) containing $150 \mathrm{mM} \mathrm{NaCl}, 0.1 \%$ Igepal, $0.1 \%$ colic acid, $2 \mu \mathrm{g} / \mathrm{ml}$ leupeptin, $2 \mathrm{mM}$ PMSF, $1 \mu \mathrm{g} / \mathrm{ml}$ pepstatin, $2 \mathrm{mM}$ EDTA and $0.1 \%$ SDS. The homogenates were centrifuged at $12,000 \times g$ for $10 \mathrm{~min}$ at $4{ }^{\circ} \mathrm{C}$ (Micro 200R, Hettich $\mathrm{GmbH}$ ). The supernatants were used for the detection of $\mathrm{OM}$. The protein concentration was determined using the bicinchoninic acid (BCA) Protein Assay Kit (Novagen $\AA$, 71285-3). A sodium dodecyl sulphate polyacrylamide gel electrophoresis (SDS-PAGE) was carried out using a $13 \%$ gel (according to the relative molecular size of $\mathrm{OM}: \approx 12 \mathrm{kDa}$ ). The proteins were electroblotted onto a nitrocellulose membrane by using the BioRad MiniProtein II System at $300 \mathrm{~mA}$ for $1 \mathrm{~h}$. The membrane was incubated with $5 \%$ non-fat dried milk dissolved in TBS-Tween for $1 \mathrm{~h}$. After washing the samples in TBS-Tween $(3 \times 5 \mathrm{~min})$, the blots were incubated with a rabbit antibody to OM (Swant, Switzerland). Preliminary experiments showed the best results at a 1:1000 working dilution. Incubation with the primary antibody was carried out in $1 \%$ non-fat dried milk in $0.1 \% \mathrm{NaN}_{3}$ overnight, at room temperature under continuous shaking. After washing the samples in TBSTween $(3 \times 5 \mathrm{~min})$, the blots were incubated with the secondary antibody (alkaline-phosphatase-conjugated anti-rabbit lgG) following the manufacturer's instructions (1:2000). After washing the samples in TBS-Tween (4x) and in TBS, the detection was performed with BCIP/NBT (5-bromo-4-chloro-3-indolyl phosphate/nitro blue tetrazolium) tablets, obtained from Sigma, in the dark room. After washing in sterile water, the membranes were dried and scanned.

\section{RNA isolation and RT-PCR}

Brains derived from mice transcardially perfused with $0.9 \% \mathrm{NaCl}$ were homogenized in $1 \mathrm{ml}$ of TRIZOL $®$ reagent per $50-100 \mathrm{mg}$ of tissue using a homogenizer (Polytron) and were then incubated for $5 \mathrm{~min}$ at RT. Chloroform $(0.2 \mathrm{ml} / \mathrm{ml}$ of TRIZOL $\otimes$ reagent) was added, tubes were vigorously shaken for $15 \mathrm{~s}$ and incubated at RT for 2-3 min. After centrifugation $\left(12,000 \times g, 15 \mathrm{~min}, 4{ }^{\circ} \mathrm{C}\right)$, the aqueous phase was transferred to a new tube and the RNA was precipitated by mixing with $0.5 \mathrm{ml}$ isopropyl alcohol. The samples were incubated at RT for $10 \mathrm{~min}$, then centrifuged $(12,000 \times \mathrm{g}, 10$ $\min , 4{ }^{\circ} \mathrm{C}$ ) and the supernatant aspired. The RNA pellet was washed once with $1 \mathrm{ml}$ ice cold $\left(-20^{\circ} \mathrm{C}\right) 75 \%$ ethanol. The RNA pellet was briefly air-dried for 5-10 min. The RNA was dissolved in $25 \mu \mathrm{l}$ RNase-free water and incubating for $10 \mathrm{~min}$ at $60^{\circ} \mathrm{C}$. The total amount of RNA was then determined by measuring the $\mathrm{OD}_{260 \mathrm{~nm}}$. For the RT-PCR reaction, total RNA $(2 \mu \mathrm{g}), 1 \mu \mathrm{l}$ random hexamer primer (Promega) and $\mathrm{H}_{2} \mathrm{O}$ yielding a total volume of 12 $\mu l$ were mixed. The samples were heated to $70{ }^{\circ} \mathrm{C}$ for $5 \mathrm{~min}$, immediately chilled on ice and briefly spun. Then the following components were added: $4 \mu \mathrm{l}$ M-MLV reaction buffer $5 x$ (Promega), $2 \mu$ ldNTP-Mix (10 mM), $1 \mu$ l recombinant RNasin $®$ ribonuclease inhibitor (Promega) and $1 \mu \mathrm{l} \mathrm{M-MLV} \mathrm{reverse} \mathrm{transcrip-}$ tase (Promega). After incubation for $60 \mathrm{~min}$ at $37^{\circ} \mathrm{C}, 2 \mu \mathrm{l}$ of the RT reaction mix were used for the PCR with the primer pairs specific for mouse OM (mOM: forward: 5'-TGA GCA TCA CGG ACA TTC TG-3'; reverse: $5^{\prime}$-ATC TTC CCA TCT CCA TCG TT-3'). The size of the resulting PCR fragment is $\approx 290 \mathrm{bp}$. The PCR amplicon was loaded on a $0.8 \%$ agarose gel. The PCR fragment was then cloned into the vector pGEM®-T Easy (Promega) and transformed into competent $E$. coli $\mathrm{Dh} 5 \alpha$. The plasmid DNA isolated from three colonies was sequenced (Microsynth $\mathrm{GmbH}$, Balgach, Switzerland).

\section{Double staining}

Double staining of cells containing OM was performed with Neurexin 1alpha/beta siRNA obtained from Santa Cruz (sc-42050).

\section{RESULTS}

In control experiments performed in young adult Wistar albino rats, PV and GABA immunoreactivity in the large calyciform terminals of the RTN was clearly discernable (Fig. 1). The
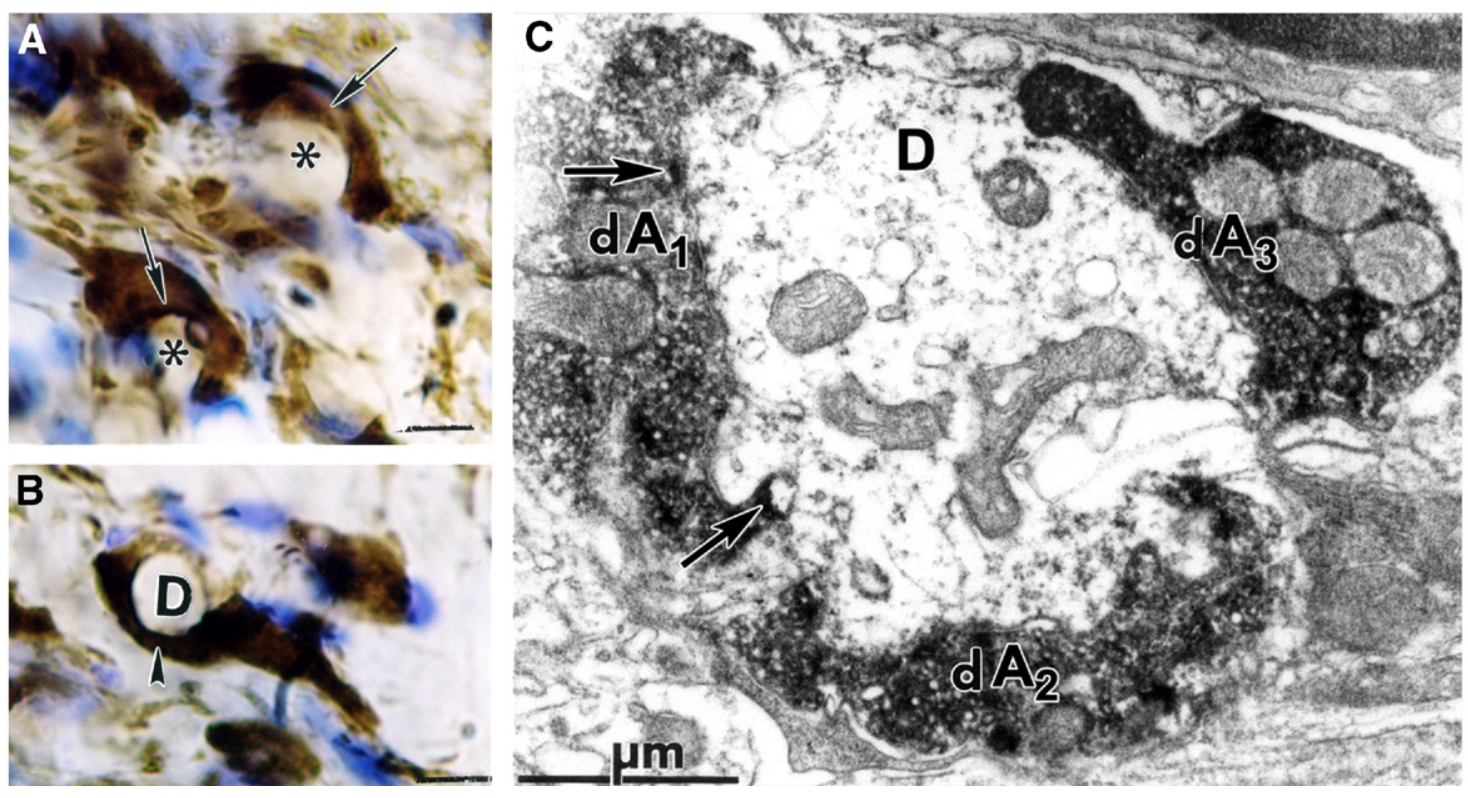

Fig. 1. Parvalbumin (PV) and GABA in large calyciform presynaptic complexes of the reticular thalamic nucleus (RTN). (A) Parvalbumin immunoreactivity in calyciform presynaptic terminals in the RTN. Arrows point at PV-immunoreactive presynaptic elements; asterisks indicate PV-negative dendritic profiles. Scale bar: $10 \mu \mathrm{m}$. (B) GABA immunoreactivity present in the same structures as in (A). D indicates dendritic profile; arrowhead indicates a calyciform GABAergic terminal. Scale bar: $10 \mu \mathrm{m}$. (C) Parvalbumin immunoreactivity evidenced at the level of electron microscopy; the dendritic profile (D) is surrounded by three PV-immunoreactive dendro-axonic elements $\left(\mathrm{dA}_{1}, \mathrm{dA}_{2}, \mathrm{dA}_{3}\right)$. For interpretation of the references to color in this figure legend, the reader is referred to the Web version of this article. 

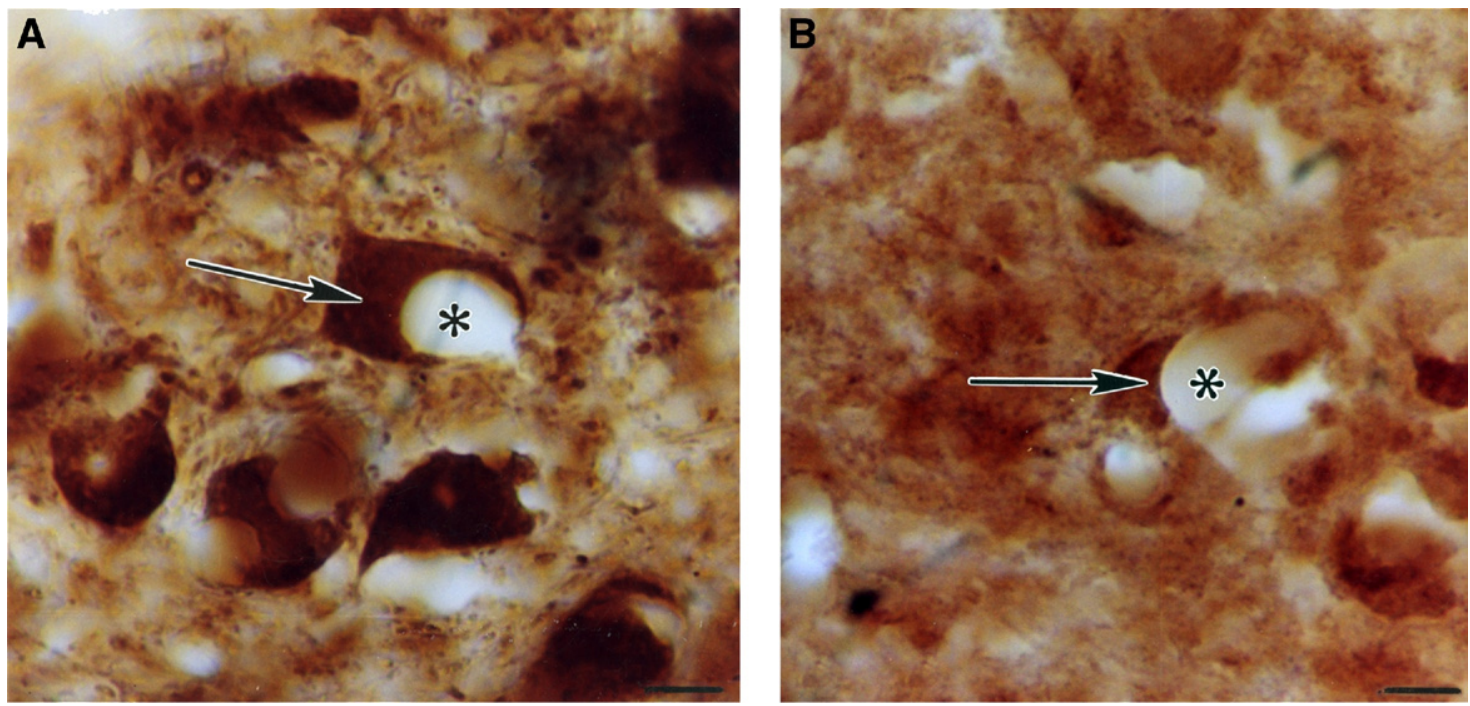

Fig. 2. Presynaptic calyciform terminals in the RTN of a C57BI/6J mouse. (A) Strong PV immunoreactivity is present in a presynaptic calyciform terminal (arrow); the asterisk indicates a PV-negative dendritic profile. Scale bar: $10 \mu \mathrm{m}$. (B) A consecutive section was stained against GABA showing strong immunoreactivity in a presynaptic calyciform terminal (arrow); the asterisk indicates a dendritic profile. Scale bar: $10 \mu \mathrm{m}$. For interpretation of the references to color in this figure legend, the reader is referred to the Web version of this article.

number of PV and GABA immunoreactive calyciform terminals was $2010 \pm 17$ in each hemisphere of the rat, as evidenced by stereological methods. Much lower numbers of calyciform terminals were observed in the RTN of C57BI/6J mice (Fig. 2); quantitative analysis revealed the presence of $262 \pm 12$ PV - and GABA-immunoreactive calyciform terminals. While immunoreactivity for GABA appeared to be unaltered in these animals (Fig. $3 A$ ), a specific immunoreaction for $\mathrm{PV}$ was absent in the gigantic calyciform terminals of $\mathrm{PV}-1-$ mice (Fig. 3B), in line with a complete absence of $\mathrm{PV}$-ir structures reported in the cortex of $\mathrm{PV}-1-$ mice (Schwaller et al., 2004). Totally unexpected, numerous, partially varicose "PV-immunoreactive" axons appeared in various parts of the diencephalon; some of them were localized in the lamina medullaris externa, surrounding the thalamus (Fig. 4A). The most likely explanation for these results was
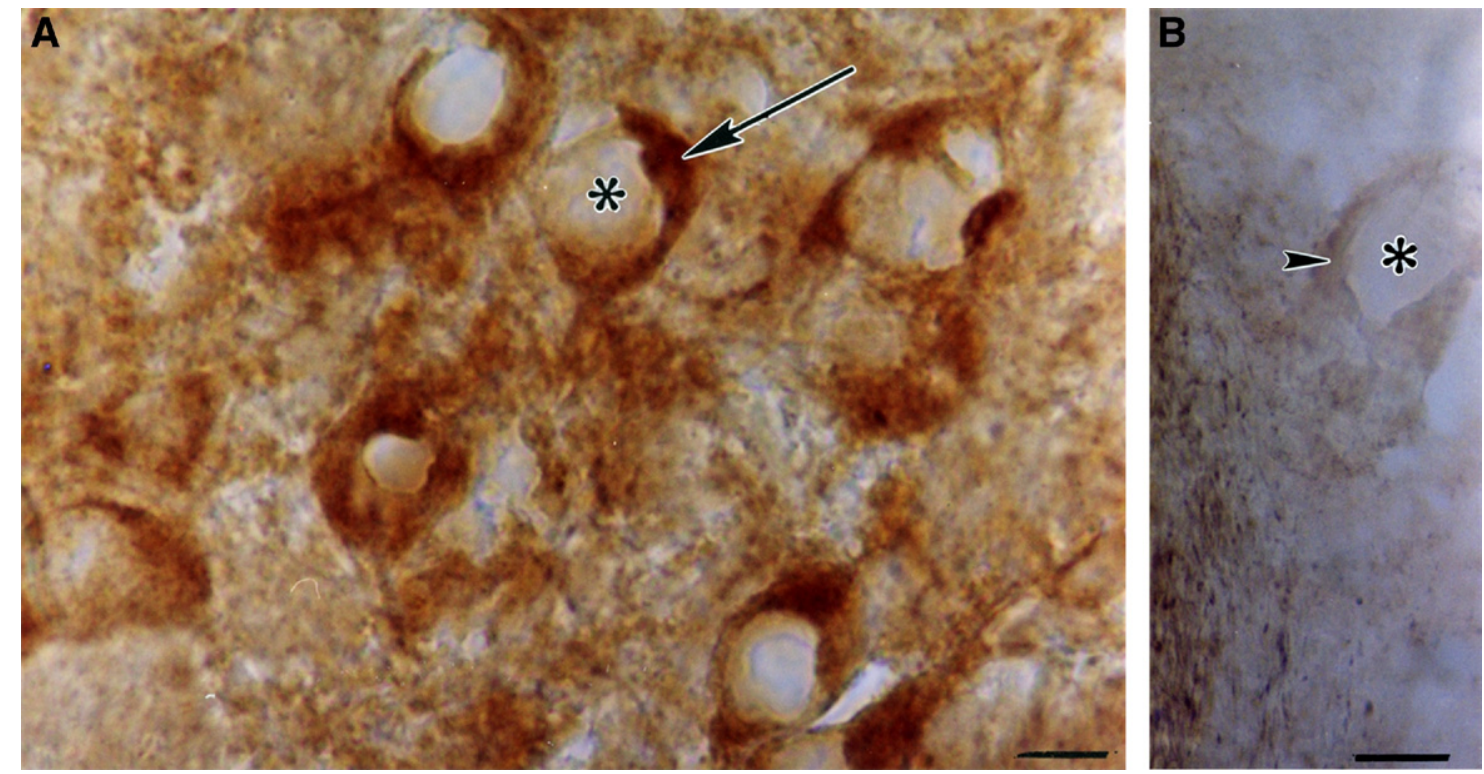

Fig. 3. Presynaptic calyciform terminals in the RTN of a PV-/- mouse. (A) GABA immunoreactivity of presynaptic calyciform terminals in the reticular thalamic nucleus of a PV-I- mouse is not different when compared to GABA-ir in a wild-type mouse (compare to Fig. 2B). An arrow points at one of the GABA-immunoreactive calyciform terminals; asterisk indicates dendritic profile. Scale bar: $10 \mu \mathrm{m}$. (B) No specific PV signal was detected in presynaptic calyciform terminals in the reticular thalamic nucleus of a PV-I- mouse. The arrowhead points at the outlines of a PV-immunonegative calyciform terminal; the faint contrast seen in this microphotograph is due to the selected parameters of microscopy (nearly closed diaphragm and lowered condenser) and is not the result of unspecific staining. The asterisk indicates a dendritic profile. Scale bar: $10 \mu \mathrm{m}$. For interpretation of the references to color in this figure legend, the reader is referred to the Web version of this article. 

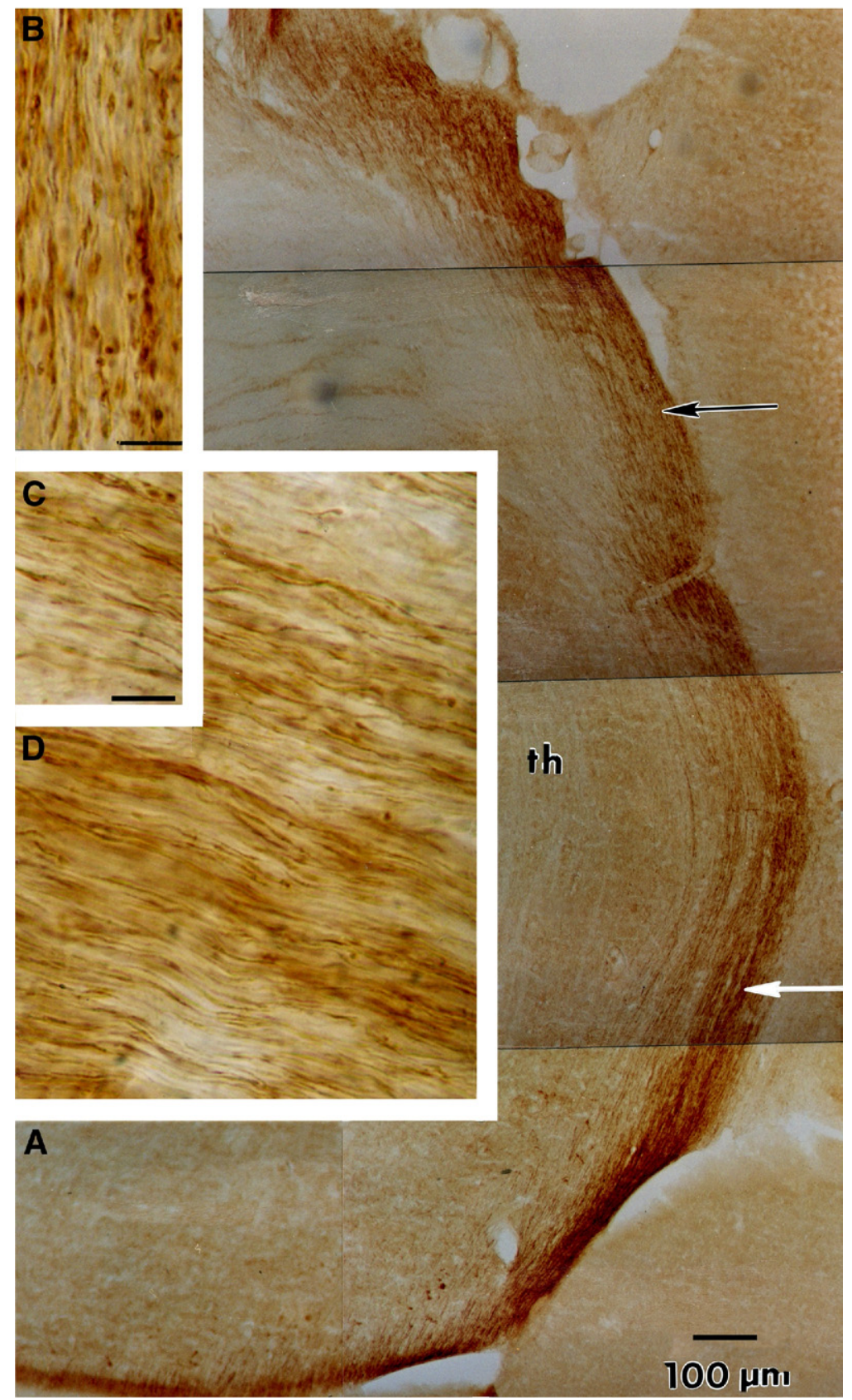

Fig. 4. (A) Bundles of oncomodulin (OM)-expressing axons (arrows) of the lamina medullaris external surrounding the thalamus (th) at low magnification in the brain of a PV $-/-$ mouse. For the immunostaining in (A-C) the OM antiserum N-19 sc-7446 (Santa Cruz) was used. Scale bar: $100 \mu \mathrm{m}$. Individual OM-expressing axons, some of them varicose (B), others lacking varicosities (C) are depicted at higher magnification. In (D), similar axonal structures can be seen in a specimen stained with the OM-specific antiserum OM3 (Swant). Scale bar for B-D: $10 \mu \mathrm{m}$. For interpretation of the references to color in this figure legend, the reader is referred to the Web version of this article.

the presence of a molecule cross-reacting with the PV antiserum. On the basis of the large similarity between parvalbumin (an alpha isoform) and OM (a beta isoform of the parvalbumin subfamily of EF-hand CaBPs, for details see Celio et al., 1996) that is $48 \%$ identity at the amino acid level, OM was considered as the prime candidate leading to the paradoxical PV staining. Thus we carried out a series of experiments to verify that the observed immunoreactivity was due to OM expression. Axonal structures, similar to those previously observed in $\mathrm{PV}-/-$ mice with the polyclonal PV antiserum were stained with the OM antibody raised against the $\mathrm{N}$-terminal $\mathrm{OM}$-specific peptide (Fig. 4B, C).

To ascertain that the observed immunoreactivity in $\mathrm{PV}-1-$ brains was due to expression of OM, a series of experiments were carried out and the results are summarized here. The immunoreactive structures were stained with OM antibodies derived from two different sources; the 

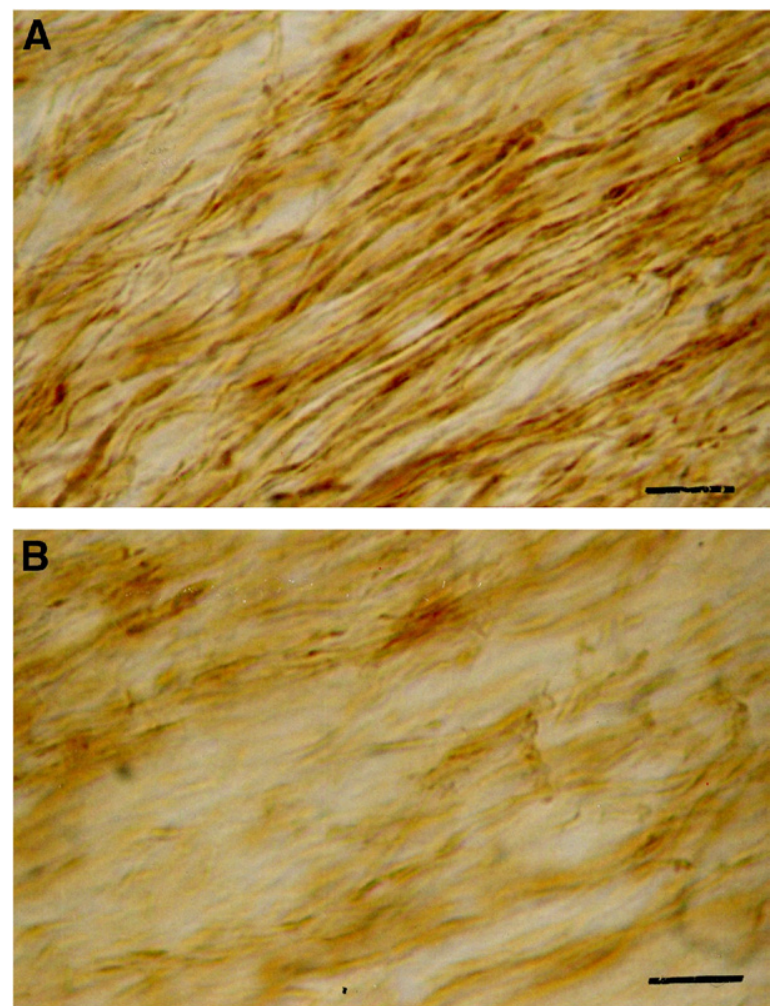

Fig. 5. (A). Bundle of OM-ir axons in specimen from a PV $-/-$ mouse stained with the OM specific peptide antibody sc-7446 (B). In a serial section pretreated with the OM blocking peptide sc-7446P, no specific immunoreaction was observed in nerve fibers. The OM immunoreactivity persisted when the serial section was incubated with the parvalbumin blocking peptide sc-7449P. Scale bar: $10 \mu \mathrm{m}$. For interpretation of the references to color in this figure legend, the reader is referred to the Web version of this article.

above-mentioned OM peptide antibody (Santa Cruz) and a polyclonal rabbit antiserum raised against full-length rat OM (Swant; for details, see Material and Methods). The axonal structures stained with the antiserum OM3 (Swant) (Fig. 4D) were asbsolutely identical to those seen with the antibody N-19 sc-7446 (Santa Cruz) for the visualization of OM (Fig. 4B, C).

Furthermore, while immunoreactivity persisted after preincubation with the PV-specific blocking peptide sc7449P (Fig. 5A), specific staining with the OM peptide antiserum $\mathrm{N}-19$ sc-7446 completely disappeared, when the primary antibody solution was preincubated with the OM-specific blocking peptide sc-7446 P (Fig. 5B).

Specific OM expression was not restricted to OM-immunopositive processes or fiber bundles, but was also detected in perikarya of cells scattered in the diencephalon (Fig. 6A-D), the cytological identity of which could not be unambiguously ascertained; accordingly, these may be neuroblasts, macrophages or possibly cells of neuroendocrine origin as well. OM appeared in the processes of these cells in a vesicular or granular form (Fig. 6A, B) resembling those reported by Yin et al. (2006). Apparently, the vesicles/granules are sometimes confined to or transformed into varicosities of nerve-fibers (Fig. 6C). OM con- taining vesicles and/or granules were present in the cellular cytoplasm of certain cells, where the immunoreactivity was accumulated in clusters of vesicles/granules within the perikaryon (Fig. 6D). In order to ascertain whether or not, OM-expressing cells belong to the cell line of neuroblasts/ neurons, double staining with the neuron-specific Neurexin 1alpha/beta siRNA was performed. In some of the OMimmunopositive cells, the OM immunoreactivity colocalized with the Neurexin staining (Fig. 6E), whereas in the majority of OM immunopositive cells Neurexin staining was not observed. Thus, this indicates that OM immunoreactivity was present in at least two different cell populations, one likely of neuronal origin (Neurexin 1alpha/beta-positive cells).

Western blot analysis of diencephalon homogenates from PV-I- mice with the OM-specific antibody (Santa Cruz) yielded a weak band of approximately $12 \mathrm{kDa}$ corresponding to the calculated $M_{r}$ of 12,260 Da for mouse OM (Fig. 7A). Furthermore, an OM-specific PCR using total RNA isolated from $\mathrm{PV}-1-$ brains resulted in a specific band of the expected size of $290 \mathrm{bp}$ (Fig. 7B). A much weaker signal was also seen with RNA isolated from the brains of $\mathrm{C} 57 \mathrm{BI} / 6 \mathrm{~J}$ wild-type mice suggesting minute amounts of OM mRNA may be also present in brains of wild-type mice.

\section{DISCUSSION}

The unchanged GABA staining in the RTN of PV - /- mice is a strong indication that $P V$ does not exert a significant control on GABA expression in the diencephalon. Evidently, subtle differences in GABA distribution and/or expression could not be determined by the methods used here and further electrophysiological analysis of the RTN of $\mathrm{PV}-1-$ mice needs to be carried out. Whether a link between GABA and calcium handling systems (e.g. PV) exists also in other parts of the central nervous system (CNS) (e.g. PMCA2 in GABAergic terminals of PV interneurons in the cortex, as reported by Burette et al., 2009, or in the amygdala, as shown by McDonald and Mascagni, 2001), where GABA colocalizes with $P V$, remains to be shown.

$\mathrm{OM}$, the beta isoform of parvalbumin, discovered by MacManus (1979) and by Brewer and MacManus (1987) was known until now to be present in adult mammals only in the organ of Corti of the inner ear, in particular in the outer hair cells (Thalmann et al., 1995; Sakaguchi et al., 1998, Hackney et al., 2005). According to our present studies, an extensive system of OM-immunoreactive axons is present in the diencephalon of PV-deficient animals. These OM-immunoreactive axons were present, inter alia, in the lamina medullaris externa of the thalamus of PVdeficient animals. Remarkably, in wild-type animals only the presence of met-enkephalin-immunopositive nerve-fibers has been reported in the lamina medullaris externa so far (Conrath et al., 1986). The fact that the OM-immunoreactive fibers had not been previously observed in wildtype mice may be due to either extremely low expression levels or the absence of any OM expression at the protein 

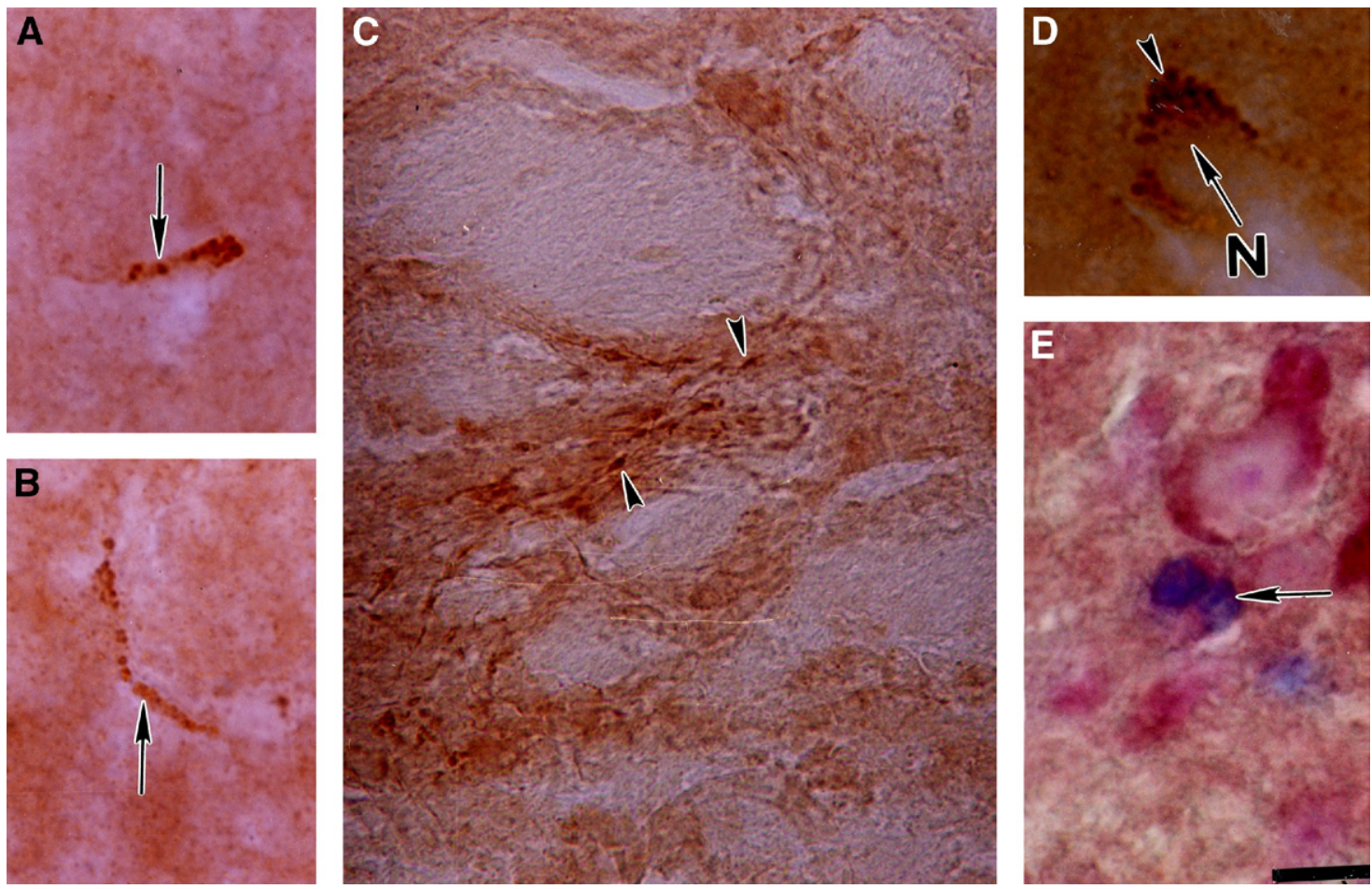

Fig. 6. (A, B): Expression of $\mathrm{OM}$ in cellular processes in the thalamus of a $\mathrm{PV}-/-$ mouse. Expression is limited to vesicles/granules (arrows) outlining the processes of the depicted cells originating from the thalamus. In (C), vesicles/granules are localized to varicosities of nerve-fibers (arrows heads), proceeding amongst immunonegative cell bodies (D): Cell body giving raise to OM immunopositive axons in the thalamus of a PV $-1-$ mouse. Arrowhead points at accumulation of OM-immunoreactive vesicles/granules in the perikaryon. $\mathrm{N}$ indicates nucleus. (E): A cell close to the hilus of the dentate gyrus. Arrow points at double staining for OM (brown) and the neuronal marker Neurexin 1alpha/beta (violet) suggesting that the cell may belong to the neuroblastic cell line. Scale bar: $10 \mu \mathrm{m}$. For interpretation of the references to color in this figure legend, the reader is referred to the Web version of this article.

level. However, our RT-PCR results indicate that also in C57BI/6J mice, low levels of OM mRNA are present in the brain. In contrast to our initial hypothesis that the neuro- transmitter (GABA) and PV could be regulated in a similar manner, we found no evidence that the absence of $P V$ in $\mathrm{PV}-1-$ mice entailed a change in GABA, since GABA

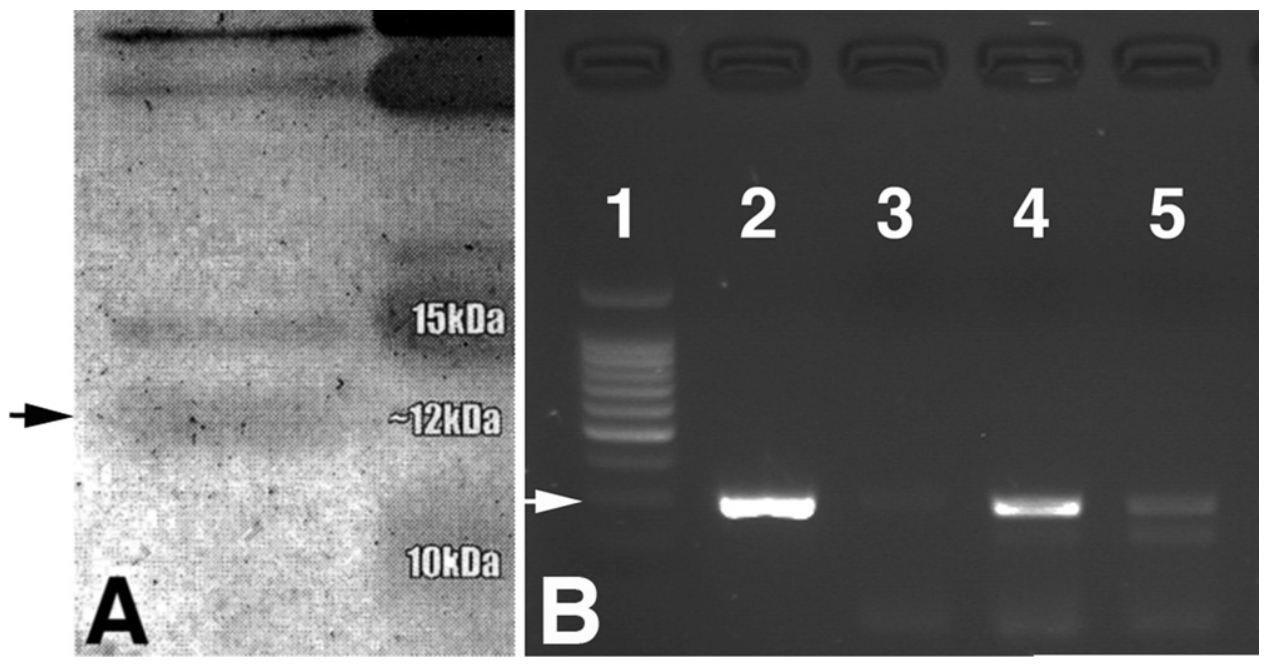

Fig. 7. (A) Western blot analysis of $\mathrm{OM}$ in a tissue homogenate isolated from the diencephalon of a PV-I- mouse. The specific band (arrow) corresponding to $\mathrm{OM}$ (left lane) has a relative molecular mass of $\approx 12 \mathrm{kDa}$ estimated from the sizes of marker proteins contained in the prestained protein ladder (right lane). The upper weaker bands in the left lane are trace amounts of the ladder proteins. (B) Agarose gel ( $0.8 \%)$ of PCR amplicons derived from reverse-transcribed RNA isolated from the diencephalons of C57BI/6J wild-type and PV-I- mice. Lanes: (1) 100 bp DNA Ladder (Promega); the position of the 300 bp band is marked by arrow. (2) positive control (290 bp) using mouse OM cDNA in vector pGEM as PCR template. (3) negative control $\left(\mathrm{H}_{2} \mathrm{O}\right.$ only, no RT product added). (4) RT-PCR product from the diencephalon of a C57-PV-/- mouse. (5) RT-PCR product from a C57BI/6J wild-type mouse. Note that a very faint band of the correct size $(290 \mathrm{bp})$ is present in samples of the diencephalon of wild-type mice. 
immunoreaction of large calyciform presynaptic terminals was essentially unaffected.

Since our initial observation of a paradoxical PV immunoreactivity in the PV $-/-$ mice was based on the crossreactivity of the applied PV antibody with its closest family member of the EF-hand family of CaBPs, namely OM, we performed several experiments to ascertain the identity of the antigen detected by the antibodies used in this study. In the report by Fu et al. (2004), the specificity of the antibody sc-7449 for alpha-PV (C-19, Santa Cruz) was demonstrated. The specificity of the antibody sc-7446 used for the detection of OM (OCM N-19, Santa Cruz) is described in the paper of Devarajan et al. (2005). And finally, the specificity of the polyclonal OM antibody OM3 (distributed by Swant, Bellinzona, Switzerland) was reported by Rentsch et al. (2006). The PV immunogen sc$7449 \mathrm{P}$ shares $56 \%$ identity with protein phosphatase 3 regulatory subunit, beta isoform: for a short peptide this homology is considered to be too low to cross-react with any known protein, since cross-reactivity is predicted to occur at $85 \%$ or greater sequence identity (L. Hatt, Santa Cruz Biotechnology, CA, USA, personal communication). The OM peptide to produce the antibody sc-7446 does not share any significant sequence identity with any known protein. Thus, the blocking peptides sc-7449P to neutralize the PV antibody and sc-7446P to neutralize the OM antibody were designed specifically for each antibody and share the same low sequence identity towards the other peptide. Also for the OM specific antibody OM3 from Swant, no crossreactivity for PV has been reported to our knowledge. In contrast, the polyclonal PV antiserum initially used in our study, cross-reacts with the beta parvalbumin, OM.

While PV is a mobile cytosolic CaBP und thus considered to be distributed homogenously throughout the cells in which it is expressed, the staining for OM observed in the brain of $\mathrm{PV}-\mathrm{I}-$ mice appeared not homogenous. OMimmunoreactivity was seen in granular/vesicular structures in cell processes (Fig. 6A, B) or in the periphery of cell bodies. Also OM-immunoreactivity in fiber bundles appeared to be relatively non-homogenous (e.g. Fig. 5A). This suggests that OM could be enclosed in compartments within these cells (vesicles/granules), which would be in line with the idea that OM may be secreted and have extracellular functions (see below). On the other hand, recent structural data on OM, most importantly calciuminduced conformational changes, indicate that this protein could also have calcium sensor functions, possibly also inside cells including nerve-fibers (for details, see Schwaller, 2009 and below). Also in situ hybridization experiments, which are now in progress using the plasmid DNA mentioned in the "Experimental procedure" chapter, and to be published in a forthcoming paper (personal communication of Prof. Karoly Gulya et al., Albert SzentGyörgyi Medical School, Szeged, Hungary) seem to strenghten our case.

Besides the nerve fibers, we also detected several OM-immunoreactive cellular elements scattered throughout the thalamus and the dentate gyrus. According to recent results, extracellular OM produced by macrophages may promote axonal elongation and regeneration (Yin et al., 2006); this theory was supported by the studies of Müller et al. (2007, 2009) and Benowitz and Yin (2008). However, more recent studies raise questions about the macrophage origin of OM (Hauk et al., 2008; Charalambous et al., 2008) and it remains to be demonstrated whether extracellular $\mathrm{OM}$ is able to promote axonal outgrowth or axonal regeneration also under normal conditions (Taylor et al., 2009). According to our studies, based on double staining with the marker Neurexin, some of the OM-expressing cells close to the gyrus dentatus of $\mathrm{PV}-\mathrm{I}-$ mice belong to the neuroblastic cell line or could even correspond to neurons. The dentate gyrus of the hippocampus is one of the brain regions, where prominent neurogenesis is reported to occur in adult mammals (Kempermann et al., 2004) although, in the view of other authors (Rakic, 2006; Breunig et al., 2007) adult neuro-neogenesis, in particular in the human neocortex, is controversial. Since changes in neurogenesis have been reported in several genetically-modified mouse strains ( $\mathrm{Li}$ and Ames, 2008; Kuhn et al., 2005), it will be challenging to see whether the selective upregulation of OM in specific cells in the dentate gyrus is connected to altered neurogenesis in $\mathrm{PV}-1-$ mice. Assuming a putative neurotrophic role of extracellular OM derived from either macrophages or from OM-expressing neurons, i.e. to induce axonal regeneration in the adult CNS, this presents an unsurpassed challenge for clinical neurologists and neurosurgeons alike.

Acknowledgments-These studies have been supported by the Hungarian Medical Research Council (ETT), grants 007/2003 and 190/2006 and the Swiss National Science Foundation (grant $310000-113518 / 1$ to B. S.). We are indebted to Mrs. Valeria Széll for technical assistance in immunohistochemical experiments and Mr. Mihály Dezsõ for computerized photomicrography.

\section{REFERENCES}

Benowitz L, Yin Y (2008) Rewiring the injured CNS: lessons from the optic nerve. Exp Neurol 209:389-398.

Breunig JJ, Arellano JI, Macklis JD, Rakic P (2007) Everything that glitters isn't gold: a critical review of postnatal neural precursor analyses. Cell Stem Cell 1:612-627.

Brewer LM, MacManus JP (1987) Detection of oncomodulin, an oncodevelopment protein in human placenta and choriocarcinoma cell lines. Placenta 8:351-363.

Burette AC, Strehler EE, Weinberg RJ (2009) "Fast" plasma membrane calcium pump PMCA2a concentrates in GABAergic terminals in the adult rat brain. J Comp Neurol 512:500-513.

Celio MR (1990) Calbindin D-28k and parvalbumin in the rat nervous system. Neuroscience 35:375-475.

Celio M, Pauls T, Schwaller B, eds. (1996) Guidebook to the calciumbinding proteins. Oxford, UK: Oxford University Press.

Charalambous P, Hurst LA, Thanos S (2008) Engrafted chicken neural tube-derived stem cells support the innate propensity for axonal regeneration within the rat optic nerve. Invest Ophthalmol Vis Sci 49:3513-3524.

Conrath M, Covenas R, Romo R, Cheramy A, Bourgoin S, Hamon M (1986) Distribution of Met-enkephalin immunoreactive fibres in the thalamus of the cat. Neurosci Lett 65:299-303.

Csillik B, Palfi A, Gulya K, Samsam M, Mihaly A, Vecsei L, KnyiharCsillik E (2002a) Parvalbumin immunoreactive large presynaptic 
complexes in the reticular thalamic nucleus of the rat. Acta Physiol Hung 89:12.

Csillik B, Palfi A, Gulya K, Mihaly A, Knyihar-Csillik E (2002b) Somatodendritic synapses in the nucleus reticularis thalami of the rat. Acta Biol Hung 53:33-41.

Csillik B, Mihaly A, Gulya K, Samsam M, Knyihar-Csillik E (2004) Parvalbumin immunoreactive calyciform presynaptic complexes in the thalamic reticular nucleus. Ann Anat 186S:40.

Csillik B, Mihaly A, Krisztin-Peva B, Chadaide Z, Samsam M, KnyiharCsillik E (2005) GABAergic parvalbumin immunoreactive large calyciform presynaptic complexes in the reticular nucleus of the rat thalamus. J Chem Neuroanat 30:17-26.

Csillik B, Mihaly A, Krisztin-Peva B, Fenyo R, Knyihar-Csillik E (2006) Calcium-binding proteins in GABAergic calyciform synapses of the reticular thalamic nucleus. Neuroreport 17:575-578.

Devarajan K, Marchant EG, Rusak B (2005) Circadian and light regulation of oxytocin and parvalbumin protein levels in the ciliated ependymal layer of the third ventricle in the C57 mouse. Neuroscience 134:539-547.

Fu YS, Shih YT, Cheng YC, Min MY (2004) Transformation of human umbilical mesenchymal cells into neurons in vitro. J Biomed Sci 11:652-660.

Gauriau C, Bernard J-F (2002) Pain pathways and parabrachial circuits in the rat. Exp Physiol 87:251-258.

Hackney CM, Mahendrasingam S, Penn A, Fettiplace R (2005) The concentrations of calcium buffering proteins in mammalian cochlear hair cells. J Neurosci 25:7867-7875.

Hauk TG, Müller A, Lee J, Schwendener R, Fischer D (2008) Neuroprotective and axon growth promoting effects of intraocular inflammation do not depend on oncomodulin or the presence of large numbers of activated macrophages. Exp Neurol 209:469-482.

Kamps MP, Sefton BM (1988) Identification of multiple novel polypeptide substrates. Oncogene 2:305-315.

Kempermann G, Jessberger S, Steiner B, Kronenberg G (2004) Milestones of neuronal development in the adult hippocampus. Trends Neurosci 27:447-452.

Kilmer W (2001) A thalamo-cortical model of the executive attention system. Biol Cybern 84:279-289.

Knyihar-Csillik E, Csillik B (2006) Plasticity of nociception: recent advances in function-oriented structural pain research. Clin Neurosci/ldeggyogy Sz 59:87-97.

Knyihar-Csillik E, Chadaide Z, Mihaly A, Krisztin-Peva B, Csillik B (2005) Effect of electrical stimulation of the reticular nucleus of the thalamus upon c-fos immunoreactivity in the retrosplenial cortex. Ann Anat 187:245-249.

Kuhn M, Shah S, Natasha T, Rittling SR (2005) A mouse model of breast cancer metastasis to the choroid of the eye. Clin Exp Metastasis 22:685-690.

Li C, Ames JB (2008) $1 \mathrm{H}, 15 \mathrm{~N}$ and $13 \mathrm{C}$ chemical shift assigments of calcium-bound calcium-binding protein 1 (CaBP'). Biomol NMR Assign 2:61-63.

MacManus JP (1979) Occurrence of a low-molecular-weight calciumbinding protein in neoplastic liver. Cancer Res 39:3000-3005.

McDonald AJ, Mascagni F (2001) Colocalzation of calcium-binding proteins and GABA in neurons of the rat basolateral amygdale. Neuroscience 105:681-693.

Müller A, Hauk TG, Fischer D (2007) Astrocyte-derived CNTF switches mature RGCs to a regenerative state following inflammatory stimulation. Brain 130:3308-3320.

Müller A, Hauk TG, Leibinger M, Marienfeld R, Fischer D (2009) Exogenous CNTF stimulates axon regeneration of retinal gan- glion cells partially via endogenous CNTF. Mol Cell Neurosci 41:233-246.

Paxinos G, Watson C (1982) The Rat Brain in stereotaxis coordinates, pp 1-82. Sydney, New York, London, Paris, San Diego, San Francisco, San Paulo, Tokyo, Toronto: Academic Press.

Pinault D (2004) The thalamic reticular nucleus. Brain Res Brain Res Rev 46:1-31.

Rakic P (2006) Neuroscience: no more cortical neurons for you. Science 313:928-929.

Rentsch JM, Hergersberg M, Banville D, Berchtold MW (2006) The LTR promoter of the rat oncomodulin gene is regulated by the cell-line specific accessibility in the LTR U3 region. Arch Biochem Biophys 447:68-79.

Rogers SW, Hughes TE, Hollmann M, Gasic GP, Deneris ES, Heinemann S (1991) The characterization and localization of the glutamate receptor subunit GluR1 in the rat brain. J Neurosci 11:27132724.

Sakaguchi N, Henzl MT, Thalmann I, Thalmann R, Schulte BA (1998) Oncomodulin is expressed exclusively by outer hair cells in the organ of Corti. J Histochem Cytochem 46:29-39.

Schwaller B (2009) The continuing disappearance of "pure" Ca2+ buffers. Cell Mol Life Sci 66:275-300.

Schwaller B, Dick J, Dhoor G, Carroll S, Vrbova G, Nicotera P, Pette D, Wyss A, Bluethmann H, Hunziker W, Celio MR (1999) Prolonged contraction-relaxation cycle of fast-twitch muscles in parvalbumin knockout mice. Am J Physiol Cell Physiol 276:C395C403.

Schwaller B, Tetko IV, Tandon P, Silveira DC, Vreugdenhil M, Henzi T, Potier MC, Celio MR, Villa AE (2004) Parvalbumin deficiency affects network properties resulting in increased susceptibility to epileptic seizures. Mol Cell Neurosci 25:650-663.

Shosaku A, Kayama Y, Sumitomo I, Sugitani M, Iwama K (1989) Analysis of recurrent inhibitory circuits in the thalamus: neurophysiology of the thalamic reticular nucleus. Prog Neurobiol 32:77-102.

Sidman RL, Angevine JB, Pierce ET (1971) Atlas of the mouse brain and spinal cord, pp 1-302. Cambridge, MA: Harvard University Press.

Steriade M (2001) The GABAergic reticular nucleus: a preferential target of corticothalamic projections. Proc Natl Acad Sci U S A 98:3625-3627.

Taylor AM, Berchtold NC, Perreau VM, Tu CH, Li Jeon N, Cotman CW (2009) Axonal regeneration in uninjured and regenerating cortical mammalian axons. J Neurosci 29:4697-4707.

Thalmann I, Shibasaki O, Comegys TH, Heinzl MT, Senarita M, Thalmann R (1995) Detection of a beta-parvalbumin isoform in the mammalian inner ear. Biochem Biophys Res Commun 215: 142-147.

Vecellio M, Schwaller B, Meyer M, Hunziker W, Celio MR (2000) Alterations in Purkinje cell spines of calbindin D-28 k and parvalbumin knock-out mice. Eur J Neurosci 12:945-954.

Villa AEP, Dutoit P, Tetko IV, Hunziker W, Celio M, Schwaller B (2000) Non-linear coupling of local field potentials across cortical sites in parvalbumin-deficient mice. Singapore: World Scientific Publishing, Pte. Ltd.

West MJ, Slomianka L, Gungersen HJ (1991) Unbiased stereological estimation of the total number of neurons in the subdivisions of the rat hippocampus using the optical fractionator. Anat Rec 231:482-497.

Yin Y, Henzl MT, Lorber B, Nakazawa T, Thomas TT, Jiang F, Langer $\mathrm{R}$, Benowitz LI (2006) Oncomodulin is a macrophage-derived signal for axon regeneration in retinal ganglion cells. Nat Neurosci 9:843-852. 